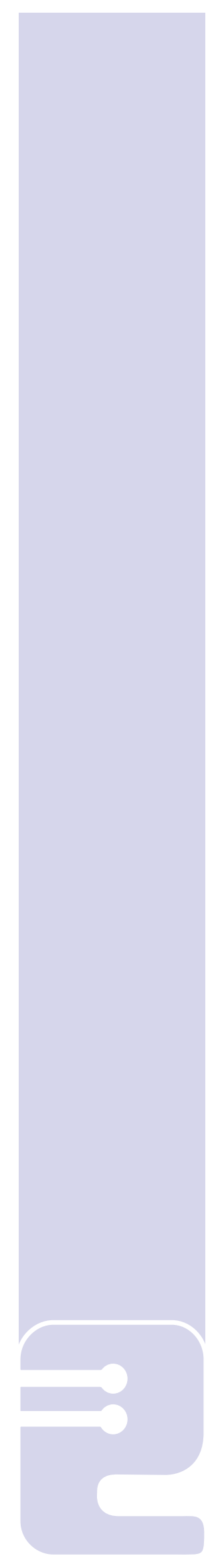

\title{
Articulación de la Robótica como Eje de Formación Interdisciplinar en el Programa de Tecnología en Electrónica de UNIMINUTO Sede Principal
}

Edgar A. Aguirre', Sergio González Gil²

Recibido: Febrero 27 de 2014 Aprobado: Junio 18 de 2014

\section{Resumen:}

Este trabajo describe la manera en que se articula el componente curricular profesional de acuerdo con las áreas de formación en el Programa de Tecnología en Electrónica de UNIMINUTO Sede Principal, usando la robótica como estrategia de aprendizaje y recurso didáctico destinado a que los estudiantes avancen en las diferentes fases de aprendizaje de las asignaturas que cursan; así mismo se refiere la experiencia del desarrollo de proyectos como metodología para apoyar los procesos de investigación, desarrollo e innovación que se registran a través de la participación de los estudiantes en actividades interinstitucionales relacionadas con la robótica. Se ha observado que los conocimientos de los estudiantes en ciencias básicas se afianzan a través de estas estrategias, mejorando su desempeño en las áreas básicas de electrónica.

Palabras clave: Robótica, electrónica, praxeologia, competencias, Semillero de robótica SERO.

\section{Abstract:}

This document shows the way to articulate the professional curricular component according the areas of study in the Electronics Technology Program of UNIMINUTO, Central Site, using robots as learning strategy and didactic resource. So the students advance in the different phases of their learning process in the courses that they assist. Also we refer the experience in projects development as methodology to support research innovation and development processes, where the students participate and also in activities with others institutions related to robotics.It has been observed that the knowledge of students in basic sciences are secured through these strategies, improving its performance in the core areas of electronics.

Keywords: Robotics, electronics, praxeology, competencies, Robotics Students Research Group - SERO.

\footnotetext{
1 Ingeniero Edgar Alirio Aguirre Buenaventura. Candidato a Magister en Ciencias de la Información y las Comunicaciones con énfasis en Teleinformática. Ingeniero en Control Electrónico e Instrumentación. Universidad Distrital Francisco José de Caldas. Actualmente se desmpeña como director de proyectos en el Parque Científico de Innovación Social - UNIMINUTO.

2 Ingeniero Sergio González Gil. Magister en administración de empresas con especialidad en proyectos, Especialista en Pedagogía y docencia Universitaria y Especialista en Telecomunicaciones Móviles. Ingeniero Electrónico. Docente de tiempo completo en el Programa de Tecnología en Electrónica - UNIMINUTO Sede Principal.
} 


\section{INTRODUCCIÓN}

El Programa de Tecnología en Electrónica de la Corporación Universitaria Minuto de Dios - UNIMINUTO Sede Principal, tiene interés en el área de robótica donde se implementan diversas plataformas por parte de los estudiantes. A partir del interés de estudiantes y docentes, que comenzaron a trabajar para compartir experiencias significativas, se formalizó posteriormente el Semillero de Robótica (SERO) en el primer semestre del año 2012, como una alternativa al trabajo académico.

Por otra parte, se ha identificado la dificultad que tienen los estudiantes para integrar sus saberes en la solución de un problema determinado, desde los conocimientos adquiridos a través de las asignaturas que toman a lo largo de la carrera.

Una estrategia para superar estas dificultades consiste en el desarrollo de proyectos en robótica, donde se combinan conocimientos diversos que permiten analizar e integrar las competencias que el estudiante debe adquirir en su proceso de formación.

El objetivo de este trabajo es evidenciar cómo las actividades desarrolladas a través del Semillero de Robótica se articulan con la formación integral de los estudiantes, permitiéndoles fortalecer su quehacer en diferentes ámbitos de la carrera. Como parte de la metodología de trabajo se identifican grupos de estudiantes que pueden participar en proyectos de robótica a través del semillero, junto con una breve descripción de los elementos de formación intergral.

\section{ASPECTOS METODOLÓGICOS PARA EL DESARROLLO DE PROYECTOS ACADÉMICOS EN ROBÓTICA}

A fin de facilitar la realización de las actividades académicas en robótica, se han agrupado los estudiantes cuatro categorías: a) quienes cursan los dos primeros períodos académicos, b) el grupo de segundo a sexto semestre, $c$ ) estudiantes de proyecto de grado de quinto y sexto semestre, y d) estudiantes de práctica profesional de cuarto, quinto y sexto semestre.

Los estudiantes de primer año cursan las asignaturas de [1] Gestión Básica de la Información, Inglés, Comunicación Escrita y Procesos Lectores, Proyecto de Vida, Precálculo, Cálculo, Probabilidad, Física, Introducción a la Tecnología, Circuitos DC, Electrónica Digital, Arquitectura de Computadores y Programación. Es aquí donde el Semillero de
Robótica SERO inicia el proceso de fortalecimiento de las temáticas de estas asignaturas por medio de actividades de introducción a la robótica, las cuales también incentivan al estudiante a participar activamente en esta área del conocimiento, ayudando a crear vínculos de redes de aprendizaje, lo cual contribuye a mejorar la permanencia de los estudiantes en el Programa; estas actividades se realizan por medio de talleres extra clase donde se exploran diferentes áreas y se experimentan ejercicios típicos de problemas de robótica.

Con el grupo de estudiantes de $2^{\circ}$ a $6^{\circ}$ semestre se siembra la inquietud por la investigación y se fortalecen los procesos investigativos, los cuales serán un eje propositivo de innovación dentro de la Universidad; es con éste grupo que se desarrollan las principa-les actividades del semillero tales como cursos, intercambios y proyectos.

Los estudiantes que cursan la asignatura de Proyecto de Grado desarrollan un proyecto de carácter tecnológico que se puede dar como parte de un semillero o grupo de investigación, al igual que los estudiantes que participan en la práctica profesional.

\subsection{Elementos de la formación integral}

Uno de los elementos centrales de las practicas pedagógicas en UNIMINUTO proviene del enfoque praxeológico, el cual se basa en la comprensión [2] de la acción humana (praxis), tanto en la acción social, entendida como el conjunto de ideas, valores, actos y palabras orientadas al desarrollo del otro (educación), como al cambio de su contexto con miras a un mejoramiento del bienestar personal y social, (Figura 1) lo cual apunta al perfil profesional del egresado.

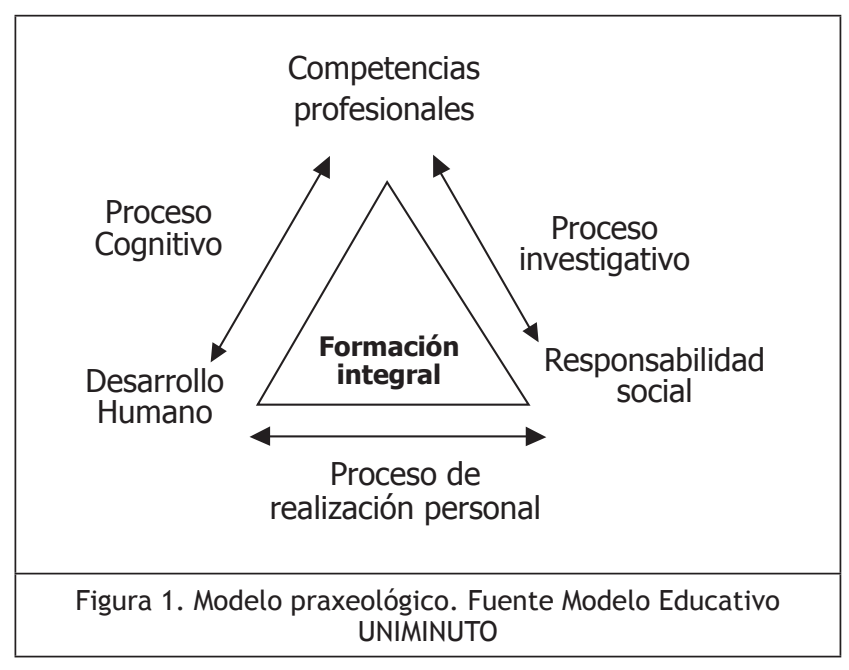


Estos elementos se articulan con los contenidos que se desarrollan en las actividades con los estudiantes; el modelo praxeológico de UNIMINUTO considera tres campos de formación los cuales son el desarrollo humano, practica social y profesional y competencias profesionales, los cuales hacen parte de la estructura curricular del Programa de Tecnología en Electrónica.

El primer campo corresponde al desarrollo humano mediante el cual se explora el proceso cognitivo y de realización personal para potencializar las cualidades de las personas, permitiéndoles adquirir las competencias adecuadas a su opción de vida, acercándolo a los demás y a su entorno de trabajo.

El segundo campo corresponde al desarrollo de responsabilidad social la cual esta expresada en una sensibilidad consciente y crítica frente a la problemática de las comunidades y del país y en unas competencias para el trabajo de promoción del desarrollo humano y social, a través del cual se logra complementar el proceso praxeológico, investigativo y de realización personal que se lleva a cabo desde las prácticas sociales y profesionales, e integra los conocimientos adquiridos en la academia con las experiencias de interacción y compromiso social suscitadas en la práctica y el contacto con las comunidades. [3]

El tercer elemento corresponde a las competencias profesionales que se obtienen a lo largo de un proceso cognitivo e investigativo que vincula la teoría y la práctica y pretende la transformación de las personas, sus conocimientos, habilidades y destrezas, generando, ante todo la actitud permanente de aprender a aprender y el hábito reflexivo, crítico e investigativo, en un contexto de interdisciplinariedad y de aplicación del conocimiento para interpretar y transformar la sociedad

\subsection{Descripción de las áreas de formación [4]} Área de gestión básica de la información GBI: El área de gestión básica de la información se enfoca en reconocer la necesidad del uso de la información y de determinar la naturaleza y nivel que esta requiere, así el estudiante da sentido a los aprendizajes adquiridos sobre herramientas tecnológicas, a partir de su correcta aplicación, utilizando el conocimiento tecnológico adquirido para la resolución de problemas académicos y de su entorno, mediante la reflexión crítica de su experiencia, para demostrar habilidades en el uso de las TIC como medio y no como fin, para compartir y construir conocimiento en entornos virtuales, mediante trabajo autónomo y colaborativo con sentido ético y responsabilidad social.

Área ciencias básicas: Utiliza el lenguaje algebraico como elemento de comunicación y realiza tratamientos adecuados en ecuaciones o sistemas de ecuaciones, así mismo modela problemas en los que intervienen variables, que se pueden relacionar de manera matemática utilizándola para resolver problemas contextualizados, creando y expresando argumentos matemáticos claros que den cuenta sobre el manejo que hacen de situaciones en el pensamiento variacional, para comprender la naturaleza de la física y la matemática, con sus limitaciones, sus interacciones con la tecnología y su papel dentro de la aplicación de leyes, teorías y modelos.

Área de electrónica análoga: Analizar y desarrollar circuitos con componentes electrónicos como los diodos y transistores (BJT, FET) para el análisis de señales de pequeña y mediana intensidad, y filtros en $A C$ y DC para el tratamiento de señales y aplicaciones básicas, también con amplificadores operacionales y elementos de potencia mediante el uso de herramientas computacionales para el análisis, diseño, construcción, simulación y ajuste de sistemas electrónicos.

Área de Circuitos: Analizar y describir el comportamiento del voltaje y la corriente en dispositivos pasivos (RLC) de acuerdo con sus características físicas tales como inductancia, capacitancia, impedancia, admitancia y potencia en voltajes AC y DC.

Área de digitales: Analizar y describir el comportamiento de las señales digitales por medio del manejo de la lógica digital para la solución de problemas, haciendo uso de la lógica secuencial y combinacional por medio de compuertas lógicas, circuitos de desplazamiento, multiplexores, demultiplexores, comparadores, circuitos de reloj y máquinas de estado, lo cual sirve de base para analizar y comprender el funcionamiento de las diferentes arquitecturas de computadores, proceso, almacenamiento y transmisión de la información para comprender la lógica de programación de bajo nivel como vhdl, assembler y otros lenguajes.

Área de control: Analizar e interpretar tratamiento de señales a través de sensores para su acondicionamiento, suministro de la información necesaria al sistema 
de control, el cual tomará decisiones basadas en modelos matemáticos y de inteligencia artificial los cuales interactúan con los actuadores los cuales pueden ser elementos mecánicos, electrónicos o neumáticos entre otros, para controlar la respuesta del sistema en diferentes tipos de ambientes.

Área de Comunicaciones: Analizar y comprender la transmisión, codificación y recepción de datos en medios guiados y no guiados, para la intercomunicación de dos o más sistemas que requieran el intercambio de información.

Área de I+D: El estudiante estará en capacidad de proponer soluciones tecnológicas que impacten significativamente en el contexto tecnológico, científico, industrial y social, haciendo uso de metodologías de investigación que le permitan desarrollar capacidades de análisis y abstracción con lo cual generar posibles soluciones a problemas reales de su entorno.

En el primer año los estudiantes adquieren capacidad de trabajo en grupo al proponer ideas, desarrollar actividades y talleres en laboratorios, donde cada individuo adquiere roles, busca y analiza información y la sintetiza, la resume y la expresa por medio de las ideas principales y secundarias extraídas de artículos científicos, libros, manuales, infografía y documentación de internet.

En el segundo año el estudiante está en la capacidad de diseñar e implementar circuitos básicos, dando solución a problemas reales sencillos soportado mediante la argumentación lógica, matemática, física, electrónica y lúdica.

En el tercer año el estudiante está en capacidad de desarrollar e implementar soluciones tecnológicas de acuerdo con el perfil de profundización elegido, argumentando técnica y tecnológicamente el diseño propuesto.

Es importante resaltar que la aplicación del modelo praxeológico ha sido el método implementado con éxito, lo que ha permitido a los estudiantes:

Ver: Relacionarse con el ambiente de trabajo, con las plataformas e identificar posibles problemas.

Juzgar: Analizar e interpretar los conocimientos propios frente a las necesidades del problema y los incentivó a la búsqueda de nuevo conocimiento y de profundizar en un tema específico.

Actuar: Confrontar los conocimientos propios frente a las necesidades reales del problema planteado, convirtiéndolos en retos los cuales fueron resolviendo gradualmente.

Devolución creativa: Desarrollar una actitud crítica que permitió generar nuevas soluciones a partir de la experiencia.

Además de lo anterior también se desarrollaron habilidades como el trabajo en equipo, el cual debe ser interdisciplinar en aspectos como:

- Áreas de la ciencia: La robótica abarca diferentes áreas para su análisis y aplicación en electrónica, el Semillero de Robótica SERO pretende ser interdisciplinario con diferentes áreas del conocimiento lo cual permitirá al estudiante interactuar con diferentes profesionales.

- Grupos de I+D, semilleros: El recurso humano se integra con otros semilleros para realizar proyectos en conjunto.

- Currículo: El trabajo de investigación integra en sus actividades las competencias y temas del proyecto curricular apoyando los procesos de los estudiantes.

En robótica los estudiantes incursionan en varios saberes los cuales se desarrollan a partir de proyectos. En el área de matemáticas específicamente en álgebra se trabajan medidas y umbrales, promedios, fracciones, relación de proporciones, unidades y conversiones, comparaciones, escalas, conjuntos numéricos, frente a las ecuaciones se desarrolla la resolución de estas, reconocimiento de variables, uso de modelos matemáticos, representación gráfica de datos con diferentes modelos, uso de modelos para explicar un concepto, uso de datos como prueba para responder preguntas. En geometría se trabajan diferentes tipos de mediciones, ubicación de coordenadas espaciales, espacios dimensionales, estadística y probabilidad, organización de datos y análisis de datos.

Como parte de la comunicación y el lenguaje se aplican estrategias para la resolución de problemas, el uso de conexiones entre las ideas y la matemática junto con el uso del lenguaje matemático para expresarse. 
En cuanto al uso de las ciencias básicas se aplican conceptos de física mecánica como el movimiento, posición, dirección y velocidad, cetro de masa, tipos de energía (calor, luz electricidad, movimiento y sonido), se analizan las propiedades de estas energías, longitudes de onda, amplitud, tono, capacidad de reflexión, color y relaciones mecánicas entre otros.

En electrónica es importante la aplicación de la robótica porque interactúa con todas las áreas de esta, el control en los procesos de decisión y de inteligencia (control ON-OFF, control no lineal, sistemas dinámicos, control óptimo, control caótico, redes neuronales, sistemas difusos etc.), En instrumentación es de gran importancia la robótica por el trabajo de sensórica y acondicionamiento de señal dado que son la percepción del mundo para el robot [5] (sensores de ultrasonido, posicionamiento, audio, vídeo etc.) y se manejan conocimientos de electrónica y circuitos.

Desde los procesos de investigación, desarrollo e innovación se trabajan todos los procesos de experimentación, planteamiento de hipótesis, búsqueda y recolección de información, análisis de datos, cálculos de error, estadística y presentación de resultados.

\section{ACTIVIDADES}

Para la evaluación de las actividades realizadas a lo largo de la formación tecnológica, el Programa de Tecnología en Electrónica de UNIMINUTO - Sede Principal ha realizado y participado en encuentros donde el estudiante pone a prueba sus conocimientos, mediante retos en tres categorías que son construcción y programación basado en Lego Mindstorm ${ }^{\circledR}$, velocista y mini sumo.

El impacto de esta propuesta se ha evidenciado con la participación de estudiantes en tres competencias desarrolladas en la primera mitad del año 2012 y dos en el año 2013, las cuales contaron con estudiantes de otras instituciones educativas tanto a nivel tecnológico como profesional, de las ciudades de Bogotá e Ibagué, permitiendo a los estudiantes del Programa tener la oportunidad de comparar los conocimientos con otros estudiantes e instituciones; se destaca la participación de dos estudiantes del Programa en un evento [6] de robótica en Guadalajara México, quedando clasificados entre los 26 mejores equipos.

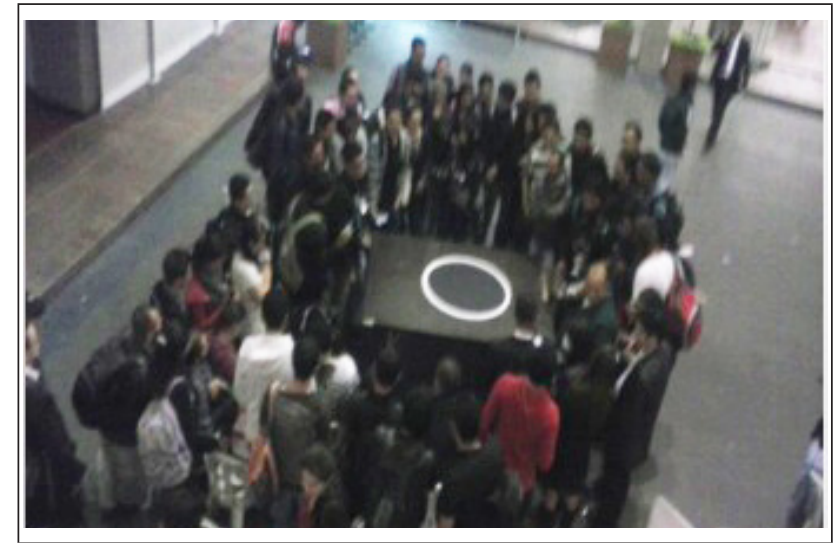

Figura 2. Imagen correspondiente al segundo encuentro de robótica, competencia de minisumo.

El encuentro desarrollado en noviembre de 2013 contó con una gran participación de estudiantes de universidades como la Central, Unibague, El Bosque, INCCA, UNIMINUTO y la Fundación CIDCA entre otros (Figura No. 2). Para este evento, se desarrolló una pista de competencia donde los concursantes diseñaron de manera libre los componentes a utilizar, a fin de completar el recorrido en el menor tiempo posible sin salirse de la pista diseñada.

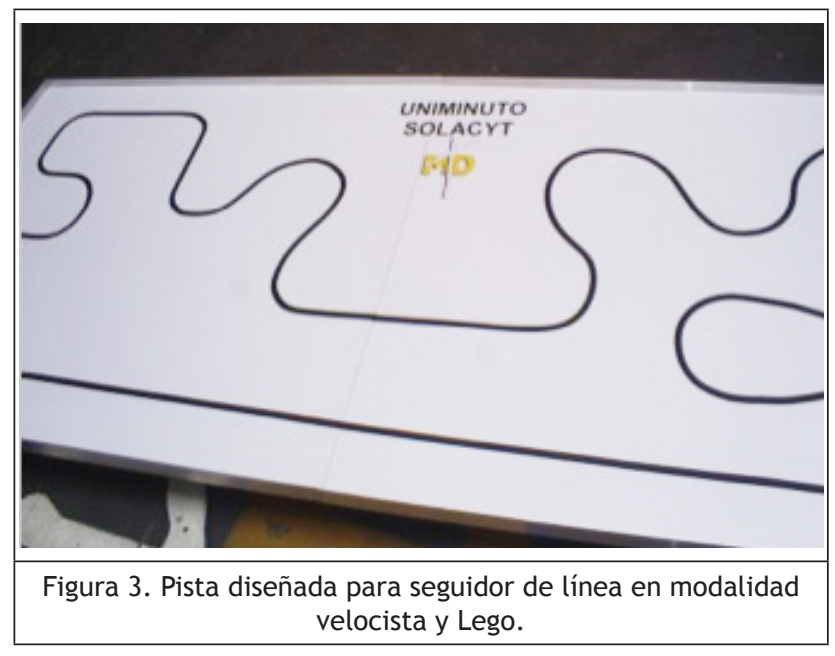

Como se observa en la Figura No. 3 la pista cuenta con dos características específicas, las cuales cuentan con unos segmentos de línea recta y curvas no tan pronunciadas, para evitar que los robots adquieran una gran velocidad y se puedan controlar los giros dados en las curvas.

Para la competencia de minisumo que contó con 11 participantes (Figura 4) se establecieron previamente las dimensiones de alto, ancho y profundidad del robot. 
La principal característica de esta competencia está en la lógica de programación que el estudiante debió haber adquirido para la detección del robot enemigo, la generación de la fuerza necesaria y evitar salir de la tabla de competencia.

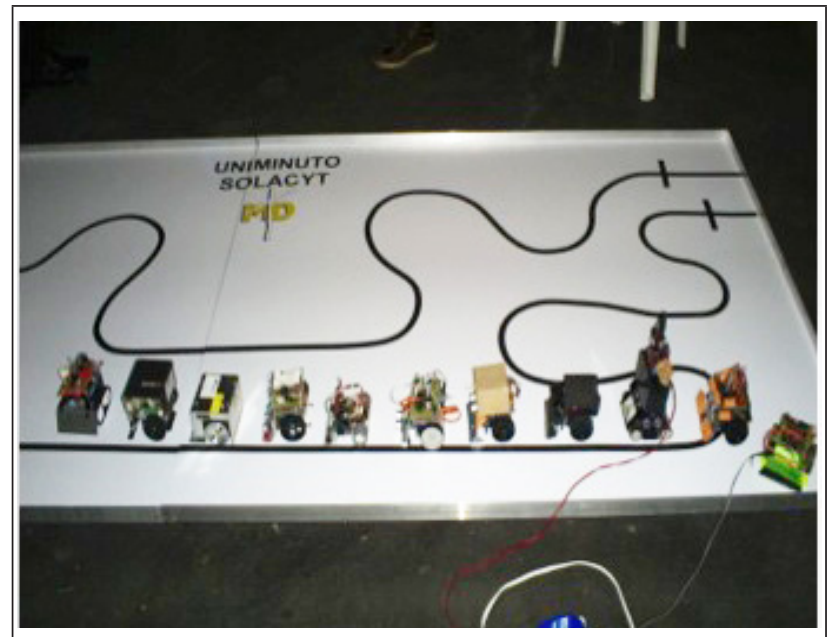

Figura 4. Vista general donde aparecen los 11 robots para la competencia de minisumo.

En la realización de estas actividades y proyectos se observa los siguiente:

Creación y diseño del robot: Una característica de los robots Lego es el refuerzo visual que genera una habilidad espacial de interpretación [7], a medida que el robot se hace más grande el estudiante debe ser capaz de interpretar las instrucciones dadas y así mismo debe ser capaz de tomar decisiones de cómo modificar la plataforma para que cumpla con los requisitos, un elemento interesante es enfocarlo desde la creencia, deseo e intención [8] para interpretar cómo debe ensamblar el robot.

Programación: igualmente la programación se realiza desde los principios de creencia, deseo e intención [8]; esto se refleja físicamente en que la creencia del mundo se interpreta por medio de los sensores, al igual que el deseo es el control del robot, las decisiones que va a tomar y finalmente la intención es la actuación, las acciones que van a cambiar el entorno del robot que se realizan por medio de motores, sonidos e imágenes.

Retos: En esta parte de la actividad el estudiante debe mejorar la programación del robot, evitando el gasto de energía y minimizando el tiempo en el procesamiento de información, toda vez que el ahorro de energía es esencial al momento de la competencia.
Al contar con la participación de otras instituciones educativas del índole local y nacional, los Tecnólogos en Electrónica de UNIMINUTO se ven forzados a mejorar su desempeño, incentivar la investigación y el deseo de superación ante los errores y fracasos presentados en la competencia.

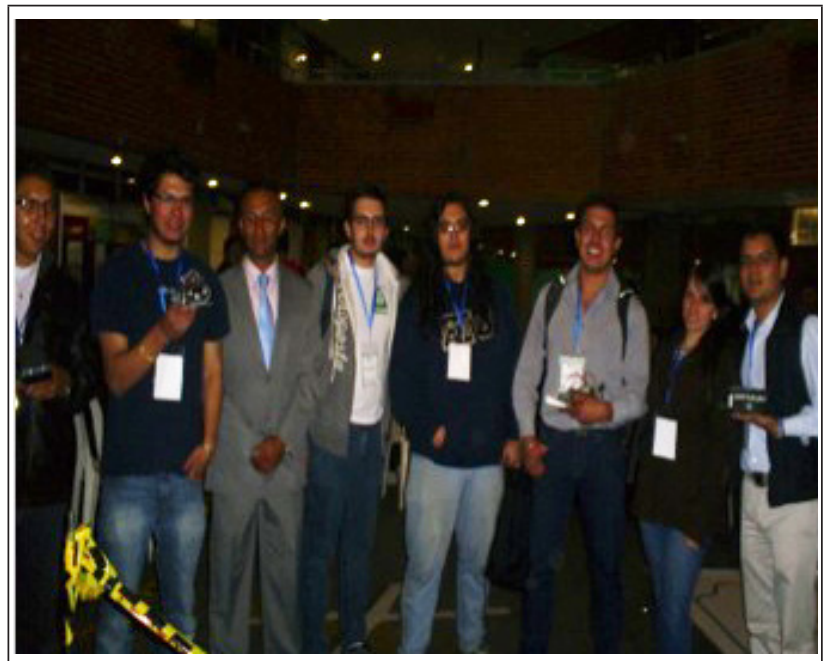

Figura 5. Foto de los Ganadores en las categorías de minisumo y velocista 30 octubre 2013.

En la Figura 5 se observa a un grupo de participante del Semillero de Robótica (SERO) del Programa de Tecnología en Electrónica de la Sede Principal de UNIMINUTO, cuyo director es el Ingeniero Carlos Herazo.

\section{CONCLUSIONES}

La propuesta del trabajo con robótica en el Programa de Tecnología en Electrónica se basa en integrar las habilidades y destrezas que los estudiantes adquieren en las diferentes asignaturas.

Se ha observado que los estudiantes aplican los conocimientos previos en ciencias básicas de una forma pŕactica, a través de los ejercicios y proyectos de clase en robótica, que se tornan en recursos didácticos, apropiándose del lenguaje y de los conceptos tales como magnitudes y medidas para representar la realidad, además se interpretan características físicas de automatismo y se manejan conceptos básicos de algoritmia [9].

Una vez afianzados estos conocimientos los estudiantes mejoran su desempeño en las áreas básicas de electrónica donde la programación de diferentes dispositivos aporta en las competencias 
de lógica, por lo que aprenden diferentes lenguajes de programación; en esta área la robótica aporta elementos importantes en la abstracción de comportamientos para ser implementados en las plataformas robóticas [10], generando competencias de trabajo en grupo para resolver problemas de competencia entre los agentes robóticos basados en las experiencias adquiridas en la resolución de ejercicios y practicas [10].

Como parte final del ciclo de aprendizaje, el estudiante puede profundizar en el área de control y comunicaciones, áreas donde enfrentan diversos métodos de control [11] como el manejo de organismos artificiales, sistemas de agentes y multiagentes, los cuales son vistos desde la perspectiva del control, para mejorar la respuesta del sistema; así mismo el modelado de los sistemas dinámicos validando los modelos teóricos propuestos [12].

\section{REFERENCIAS BIBLIOGRÁFICAS}

1. Modelo curricular, para programas tecnológicos y universitarios, versión 4.1, Corporación Universitaria UNIMINUTO Vicerrectoría académica-sede principal, Bogotá, julio 26 de 2009

2. Plan de desarrollo 2008 - 2012 SP UNIMINUTO.

3. Guía para la elaboración del proyecto curricular del programa PCP. 2011 Corporación Universitaria Minuto de Dios Vicerrectoria Académica.

4. PCP proyecto curricular de tecnología en electrónica. Corporación universitaria minuto de Dios.

5. Un enfoque basado en competencias de sistemas multiagentes para la enseñanza de Inteligencia Artificial, Cecchi L, et al. REVISTA TE \& ET 5 edición. 2010. Grupo de Investigación en
Robótica Inteligente Departamento de Ciencias de la Computación Facultad, de Economía y Administración Universidad Nacional del Comahue, Neuquén, ARGENTINA

6. Loaiza L, 2014, Marzo, Robot de minisumo de Uniminuto entre los 16 mejores en Robomatrix 2014, [Online]. Disponible en: http://www. uniminuto.edu/web/facultad-de-ingenieria/- / robot-de-minisumo-de-uniminuto-entre-los-16mejores-en-robomatrix-2014.

7. Generation NXT: Building Young Engineers With LEGOs, Tanja Karp, Senior Member, IEEE, Richard Gale, Senior Member, IEEE. Laura A. Lowe, Vickie Medina, and Eric Beutlich, IEEE TRANSACTIONS ON EDUCATION, VOL. 53, NO. 1, february 2010

8. Wooldridgie, M. \& Jennings, R. MIT(Ed.) Multiagent System a modern Approach to Distribued Artificial Intelligence. The MIT press, 1999

9. J. lerache, M. Bruno, M. Dittler, and N. Mazza, "Robots y Juguetes Autónomos una Oportunidad en el Contexto de las Nuevas Tecnologías en Educación", in Proc. JIISIC, 2008, pp.371-380.

10. Franklin E, "Teaching teachers beyond the tool: incorporating robotics and data collection into middle and high schools" en Conf. Rec. 2008 Annual Conference \& Exposition. Engineering Professional Development for K12 Teachers.

11. Miglino, O., Lund, H., y Cardaci, M. (1999). La robótica como herramienta para la educación. Journal of Interactive Learning Research, 10(1), 25-47.

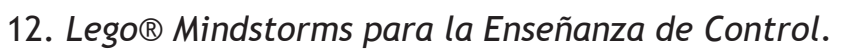
Ballesteros M, Cotrino C. Departamento de Ingeniería Electrónica Pontificia Universidad Javeriana, Bogotá, Colombia 2010. 